\title{
GLUCOSIDES AND UREA DERIVATIVES FROM THE SEEDS OF SCAPHIUM MACROPODUM (MIQ.) BEUMÉE
}

\author{
Vu Anh Tu ${ }^{1}$, Chau Ngoc Diep ${ }^{1}$, Nguyen Huu Toan Phan ${ }^{2, *}$, Nong Van Duy ${ }^{2}$, \\ Nguyen Thi Dieu Thuan ${ }^{2}$, Nguyen Van Thanh ${ }^{1}$, Nguyen Xuan Cuong', \\ Nguyen Hoai Nam ${ }^{1}$, Chau Van Minh ${ }^{1}$

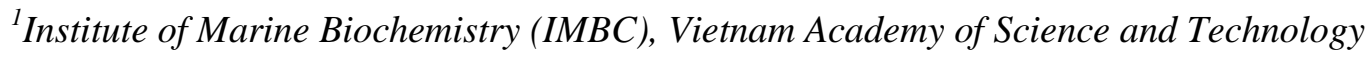 \\ (VAST), Hanoi, Vietnam \\ ${ }^{2}$ Tay Nguyen Institute for Scientific Research, VAST, Da Lat city, Vietnam \\ *Email: nhtphan@gmail.com
}

Received: 28 July 2015; Accepted for publication: 2 January 2016

\begin{abstract}
Five known compounds \{carbonylbis[imino(6-methyl-3,1-phenylene)]\}bis[carbamic acid] dimethyl ester (1), (1'R,3'S,5'R,8'S,2E,4E)-dihydrophaseic acid 3'- $O$ - $\beta$-D-glucopyranoside (2), 3methylbutan-1-ol $\beta$-D-glucopyranoside (3), astragalin (4) and daucosterol (5) were isolated from the methanol extract of the seeds of Scaphium macropodum (Miq.) Beumée. The structures of the isolated compounds were elucidated by spectroscopic methods including NMR and MS, and also by comparison with the literature data. Compounds 1-3 were isolated from this plant for the first time.
\end{abstract}

Keywords: Scaphium macropodum, Sterculiaceae, glucosides, urea derivative.

\section{INTRODUCTION}

Scaphium macropodum (Miq.) Beumée ex K. Heyne (Sterculiaceae) also known as malva nut (English) or uoi (Vietnam), is a large tree up to $30 \mathrm{~m}$ in height. They are widely distributed in tropical rainforests in Vietnam, Myanmar, Cambodia, Thailand, Malaysia and Indonesia. The seeds of this plant have been used in Vietnam for the treatment of heatdisease, dry cough, sore throat, toothache, sore redeyes and dysentery. It also has cooling properties [1]. The chemical constituents of the seeds and the stem bark of this plant such as alkaloids, cerebrosides, flavonoids, triterpenes, steroids and sesquiterpenes have been reported [2-4]. In this article, we report the isolation and structural elucidation of five known compounds 1-5 from the seeds of S.macropodum in Vietnam. Compounds 1-3 have not been previously isolated from this species.

\section{EXPERIMENTAL}

\subsection{General experimental procedures}


The ESI-MS was measured on Agilent 1260 series single quadrupole LC/MS systems. NMR spectra were recorded on a Bruker AM500 FT-NMR spectrometer (Bruker, Billerica, MA, U.S.A.) using TMS as an internal standard. Column chromatography (CC) was performed using a silica gel (Kieselgel 60, 70-230 mesh and 230-400 mesh, Merck, Darmstadt, Germany) or YMC RP-18 resins (30 - $50 \mu \mathrm{m}$, Fuji Silysia Chemical Ltd, Aichi, Japan). Thin layer chromatography (TLC) used pre-coated silica gel $60 \mathrm{~F}_{254}$ (1.05554.0001, Merck, Darmstadt, Germany) and RP-18 $\mathrm{F}_{254 \mathrm{~s}}$ plates (1.15685.0001, Merck, Darmstadt, Germany) and compounds were visualized by spraying with aqueous $10 \% \mathrm{H}_{2} \mathrm{SO}_{4}$ and heating for 3-5 minutes.

\subsection{Plant material}

The samples of the plant Scaphium macropodum (Miq.) Beumée were collected in May 2013 at Da Huoai, Lam Dong and identified by Dr. Nong Van Duy from the Tay Nguyen Institute for Scientific Research, VAST. A voucher specimen (No. TN3/309) was deposited at the Tay Nguyen Institute for Scientific Research, VAST.

\subsection{Extraction and isolation}

The air dried and powdered seeds of $S$. macropodum $(4.5 \mathrm{~kg})$ were extracted with methanol at $40^{\circ} \mathrm{C}$ three times. Methanolic extracts were combined and evaporated under vacuum. This extract $(500 \mathrm{~g})$ was suspended in water and partitioned in turn with $n$-hexane, $\mathrm{CH}_{2} \mathrm{Cl}_{2}$, and EtOAc to provide the corresponding extracts: $n$-hexane $(\mathrm{H}, 80 \mathrm{~g}), \mathrm{CH}_{2} \mathrm{Cl}_{2}(\mathrm{D}, 7.5 \mathrm{~g})$, EtOAc $(\mathrm{E}$, $3.5 \mathrm{~g}$ ) and a water layer.

The D extract was subjected to silica gel CC using stepwise elution of n-hexane/acetone (from 10:0 to 0:10) to afford 8 fractions D1 - D8, respectively. Fraction D6 was further separated by silica gel CC eluting with $\mathrm{CH}_{2} \mathrm{Cl}_{2} / \mathrm{MeOH}$ (40:1) to give compound $\mathbf{1}(10 \mathrm{mg})$.

The E extract was chromatographed over a column of Sephadex LH-20, eluting with $\mathrm{MeOH} / \mathrm{H}_{2} \mathrm{O}(1: 1)$ to afford six fractions (E1-E6). Fraction E6 $(155 \mathrm{mg})$ was further purified YMC RP-18 CC eluting with $\mathrm{MeOH} / \mathrm{H}_{2} \mathrm{O}$ (1:1) to give compounds 5 (12 mg) and 4 (6 mg). $\mathrm{T} 1$. Positive ESI-MS $m / z 387[\mathrm{M}+\mathrm{H}]^{+}$.

(1'R,3'S,5' $\left.R, 8^{\prime} S, 2 E, 4 E\right)$-dihydrophaseic acid $3^{\prime}-O-\beta$-D-glucopyranoside (2): Colorless amophous powder; ${ }^{1} \mathrm{H}-\mathrm{NMR}\left(500 \mathrm{MHz}, \mathrm{CD}_{3} \mathrm{OD}\right)$ and ${ }^{13} \mathrm{C}-\mathrm{NMR}\left(125 \mathrm{MHz}, \mathrm{CD}_{3} \mathrm{OD}\right)$ (see Table 2). Positive ESI-MS $m / z 467[\mathrm{M}+\mathrm{Na}]^{+}$.

3-methylbutan-1-ol $\beta$-D-glucopyranoside (3): Colorless needles; ${ }^{1} \mathrm{H}-\mathrm{NMR}$ (500 $\mathrm{MHz}$, $\left.\mathrm{CD}_{3} \mathrm{OD}\right) \delta 3.96(1 \mathrm{H}, \mathrm{m}, \mathrm{H}-1 \mathrm{a}), 3.59(1 \mathrm{H}, \mathrm{m}, \mathrm{H}-1 \mathrm{~b}), 1.54(2 \mathrm{H}, \mathrm{dd}, J=7.0, J=13.5 \mathrm{~Hz}, \mathrm{H}-2), 1.77$ (1H, m, H-3), 0.95 (3H, s, H-4), 0.93 (3H, s, H-5), 4.26 (1H, d, J = 8.0 Hz, H-1'), 3.18 (1H, dd, $\left.J=8.0, J=9.0 \mathrm{~Hz}, \mathrm{H}-2^{\prime}\right), 3.36$ (1H, t, $\left.J=8.5 \mathrm{~Hz}, \mathrm{H}-3^{\prime}\right), 3.31$ (1H, m, H-4'), 3.27 (1H, m, H-5'), $3.69(1 \mathrm{H}, \mathrm{dd}, J=5.5, J=12.0 \mathrm{~Hz}, \mathrm{H}-6$ ' a $)$, and $3.89\left(1 \mathrm{H}, \mathrm{dd}, J=2.0, J=12.0 \mathrm{~Hz}, \mathrm{H}-6{ }^{\prime} \mathrm{b}\right) ;{ }^{13} \mathrm{C}-$ NMR (125 MHz, CD 3 OD) $\delta 22.98$ (C-5), 23.02 (C-4), 26.06 (C-3), 39.66 (C-2), 62.81 (C-6'), 69.28 (C-1), 71.71 (C-4'), 75.14 (C-2'), 77.92 (C-5'), 78.17 (C-3'), and 104.41 (C-1'). Positive ESI-MS $m / z 251[\mathrm{M}+\mathrm{H}]^{+}$.

Astragaline (4): Yellow powder. ${ }^{1} \mathrm{H}-\mathrm{NMR}\left(500 \mathrm{MHz}, \mathrm{CD}_{3} \mathrm{OD}\right) \delta 6.18(1 \mathrm{H}, \mathrm{d}, J=2.0 \mathrm{~Hz}$, H-6), 6.37 (1H, d, $J=2.0 \mathrm{~Hz}, \mathrm{H}-8), 7.93\left(2 \mathrm{H}, \mathrm{d}, J=8.5 \mathrm{~Hz}, \mathrm{H}-2^{\prime}, \mathrm{H}-6^{\prime}\right), 6.90(2 \mathrm{H}, \mathrm{d}, J=8.5 \mathrm{~Hz}$, H-3', H-5'), $5.47\left(1 \mathrm{H}, \mathrm{d}, J=7.5 \mathrm{~Hz}, \mathrm{H}-1^{\prime \prime}\right), 4.32\left(1 \mathrm{H}, \mathrm{m}, \mathrm{H}-2^{\prime \prime}\right), 3.90\left(1 \mathrm{H}, \mathrm{m}, \mathrm{Hz}, \mathrm{H}-3^{\prime \prime}\right), 3.82$ $\left(1 \mathrm{H}, \mathrm{m}, \mathrm{H}-4^{\prime \prime}\right), 3.48\left(1 \mathrm{H}, \mathrm{m}, \mathrm{H}-5^{\prime \prime}\right), 3.80\left(1 \mathrm{H}, \mathrm{dd}, J=2.5, J=12.0 \mathrm{~Hz}, \mathrm{H}_{\mathrm{a}}-6^{\prime \prime}\right)$, and $3.55(1 \mathrm{H}, \mathrm{dd}$, $\left.J=4.5, J=12.0 \mathrm{~Hz}, \mathrm{H}_{\mathrm{b}}-6{ }^{\prime \prime}\right) ;{ }^{13} \mathrm{C}-\mathrm{NMR}\left(125 \mathrm{MHz}, \mathrm{CD}_{3} \mathrm{OD}\right) \delta: 158.51$ (C-2), 135.46 (C-3), 179.53 (C-4), 163.09 (C-5), 99.87 (C-6), 165.97 (C-7), 94.74 (C-8), 159.09 (C-9), 105.75 (C- 
10), 122.80 (C-1'), 132.27 (C-2', C-6'), 116.07 (C-3', C-5'), 161.56 (C-4'), 104.07 (C-1"), 75.74 (C-2"), $78.05\left(\mathrm{C}-3^{\prime \prime}\right), 71.37\left(\mathrm{C}-4^{\prime \prime}\right), 78.43\left(\mathrm{C}-5^{\prime \prime}\right)$, and $62.64\left(\mathrm{C}-6^{\prime \prime}\right)$. Positive ESI-MS $\mathrm{m} / \mathrm{z} 471$ $[\mathrm{M}+\mathrm{Na}]^{+}$.

Daucosterol (5): White crystals.

\section{RESULT AND DISCUSSION}<smiles>COC(=O)Nc1cc(NC(=O)Nc2ccc(C)c(NC(=O)OC)c2)ccc1C</smiles>

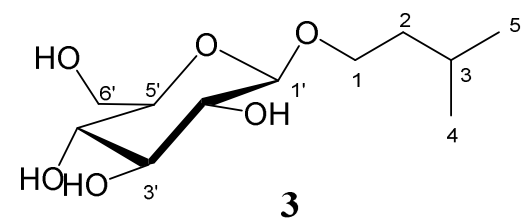

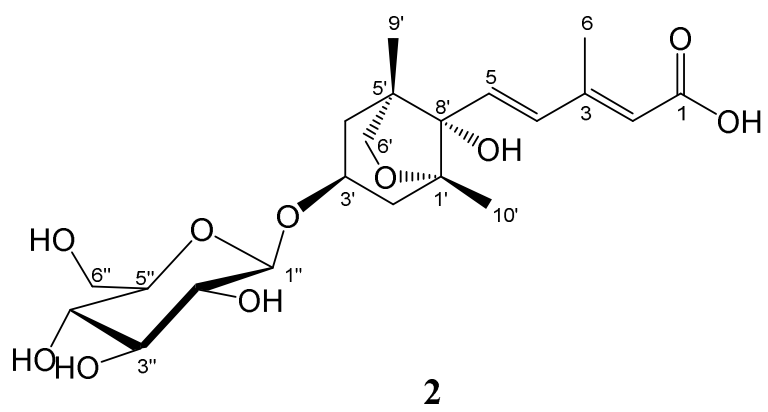<smiles>O=C1C2=C(O)C(O)=C(O)C(O)=C(O2)OC(c2ccc(O)cc2)=C1Oc1c(O)cc(O)cc1O</smiles>

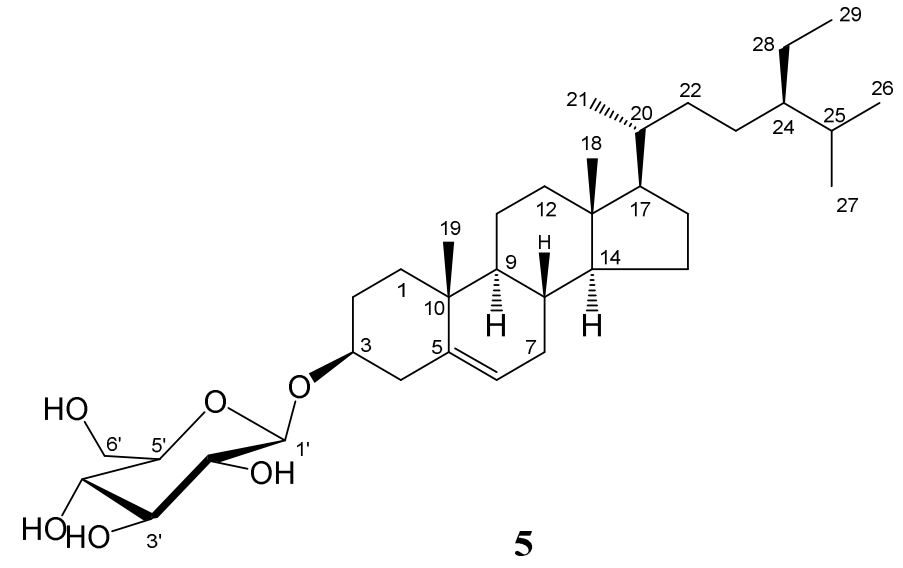

Figure 1. Structure of compounds $\mathbf{1} \mathbf{- 5}$.

Compound 1 (Fig. 1) was obtained as white powder. Its molecular formula was established as $\mathrm{C}_{19} \mathrm{H}_{22} \mathrm{~N}_{4} \mathrm{O}_{5}$ on the basis of a ion peak [M + H] $]^{+}$at $\mathrm{m} / z, 387$ in ESI-MS. The ${ }^{13} \mathrm{C}$ and DEPT NMR spectra showed only 10 resonance signals, including two amide carbonyls $\left(\delta_{\mathrm{C}} 152.4\right.$ and 154.74), one methyl, one methoxy, three $\mathrm{sp}^{2}$ methynes and three $\mathrm{sp}^{2}$ quaternary carbons, which suggested that 1 might have a symmetrical structure. The ${ }^{1} \mathrm{H}$ NMR spectrum displayed signals for an ABX coupling system at $\delta_{\mathrm{H}} 7.50\left(1 \mathrm{H}, \mathrm{br} \mathrm{s}, \mathrm{H}-2, \mathrm{H}^{\prime} 2^{\prime}\right), 7.13(1 \mathrm{H}, \mathrm{dd}, J=2.0, J=8.0 \mathrm{~Hz}, \mathrm{H}-$ 4, H-4'), $7.05\left(1 \mathrm{H}, \mathrm{d}, J=8.0 \mathrm{~Hz}, \mathrm{H}-5, \mathrm{H}-5^{\prime}\right)$ indicating the typical 1,3,4-trisubstituted benzene ring. The structure of benzene ring was further established by HMBC correlations (Fig. 2) between $\mathrm{H}-2$ and C-3, C-4, C-6, and between $\mathrm{H}-4$ and C-2, C-3, C-6, and between H-5 and C-1, $\mathrm{C}-3$. Moreover, two singlet signals at $\delta_{\mathrm{H}} 8.50(1 \mathrm{H})$ and $8.77(1 \mathrm{H})$ were assignable to two amine group, which were attached to $\mathrm{C}-3$ and $\mathrm{C}-1$ respectively, on the basis of the long-range correlations from proton 3-NH to C-2, C-3, C-4, carbon NHCONH, and from proton 1-NH to C$1, \mathrm{C}-2, \mathrm{C}-6$. In the aliphatic region, a singlet at $\delta_{\mathrm{H}} 2.12(3 \mathrm{H})$ and an other singlet at $\delta_{\mathrm{H}} 3.65(3 \mathrm{H})$ 
were assigned to methyl group 6-Me and methyl ester group respectively, due to the HMBC cross peaks from proton $6-\mathrm{Me}$ to $\mathrm{C}-1, \mathrm{C}-5, \mathrm{C}-6$ and from proton OMe to carbon NHCOO. On the basis of the above evidence, the structure of 1 was identified as \{carbonylbis[imino(6methyl-3,1-phenylene)]\} bis[carbamic acid] dimethyl ester by comparison of spectral data with those reported in the literature [5].

Table 1. The NMR data of compound $\mathbf{1 .}$

\begin{tabular}{|c|c|c|c|c|c|c|c|}
\hline $\mathbf{C}$ & ${ }^{{ }^{*}} \boldsymbol{\delta}_{\mathbf{C}}{ }^{\mathbf{a}}$ & $\boldsymbol{\delta}_{\mathbf{C}}{ }^{\mathbf{a}, \mathbf{b}}$ & $\boldsymbol{\delta}_{\mathbf{H}}{ }^{\mathbf{a}, \mathbf{c}}$ & $\mathbf{C}$ & ${ }^{{ }^{*}}{\boldsymbol{\delta}_{\mathbf{C}}}^{{ }^{\mathbf{a}}}$ & $\boldsymbol{\delta}_{\mathbf{C}}{ }^{\mathbf{a}, \mathbf{b}}$ & $\boldsymbol{\delta}_{\mathbf{H}}{ }^{\mathbf{a}, \mathbf{c}}$ \\
\hline $1,1^{\prime}$ & 136.7 & 136.48 & - & $\mathrm{Me}-6$ & 17.3 & 17.07 & $2.12(3 \mathrm{H}, \mathrm{s})$ \\
\hline $2,2^{\prime}$ & 114.6 & 114.38 & $7.50(1 \mathrm{H}, \mathrm{br} \mathrm{s})$ & $\mathrm{MeO}$ & 51.8 & 51.62 & $3.65(3 \mathrm{H}, \mathrm{s})$ \\
\hline $3,3^{\prime}$ & 137.9 & 137.72 & - & $\mathrm{NHCONH}$ & 152.6 & 152.43 & - \\
\hline $4,4^{\prime}$ & 115.0 & 114.79 & $7.13(1 \mathrm{H}, \mathrm{dd}, 2.0 ; 8.0)$ & $\mathrm{NHCOO}$ & 154.9 & 154.74 & - \\
\hline $5,5^{\prime}$ & 130.5 & 130.29 & $7.05(1 \mathrm{H}, \mathrm{d}, 8.0)$ & $\mathrm{N} \underline{\mathrm{HCONH}} \underline{-}$ & - & $8.50(1 \mathrm{H}, \mathrm{s})$ \\
\hline $6,6^{\prime}$ & 124.8 & 124.64 & - & $\mathrm{N} \underline{\mathrm{HCOO}}$ & - & - & $8.77(1 \mathrm{H}, \mathrm{s})$ \\
\hline
\end{tabular}

${ }^{a}$ recorded in $\mathrm{DMSO}-d_{6},{ }^{b} 125 \mathrm{MHz},{ }^{c} 500 \mathrm{MHz}$,

${ }^{\#} \delta_{C}$ of \{carbonylbis[imino(6-methyl-3,1-phenylene)]\}bis[carbamic acid] dimethyl ester[5]
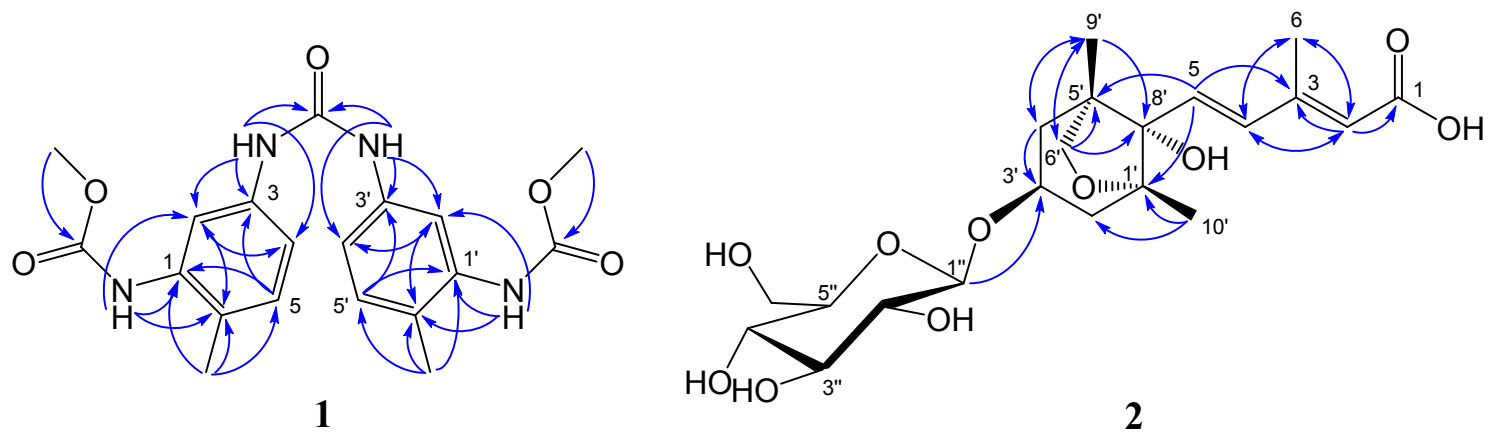

Figure 2. Key HMBC correlations of compounds 1-2.

Compound 2 (Fig. 1) was isolated as colorless amorphous powder. A molecular formula of $\mathrm{C}_{21} \mathrm{H}_{32} \mathrm{O}_{10}$ was determined for compound 2 on the basis of the observation of a molecular ion peak $[\mathrm{M}+\mathrm{Na}]^{+}$at $\mathrm{m} / z 467$ in ESI-MS. The ${ }^{1} \mathrm{H}$ and ${ }^{13} \mathrm{C}$ NMR spectra in combination with the HSQC spectrum of 2 exhibited signals for an acid carbonyl group at $\delta_{\mathrm{C}} 177.20$, a tertiary methyl group at $\delta_{\mathrm{H}} 2.03(3 \mathrm{H}, \mathrm{s}, \mathrm{H}-6) / \delta_{\mathrm{C}} 20.82$, two trans olefinic signals at $\delta_{\mathrm{H}} 7.88(1 \mathrm{H}, \mathrm{d}, J=16.0 \mathrm{~Hz}$, $\mathrm{H}-4) / \delta_{\mathrm{C}} 132.63$ and $\delta_{\mathrm{H}} 6.37(1 \mathrm{H}, \mathrm{d}, J=16.0 \mathrm{~Hz}, \mathrm{H}-5) / \delta_{\mathrm{C}} 132.02$, one trisubstituted double bond signal at $\delta_{\mathrm{H}} 5.85(1 \mathrm{H}$, br s $) / \delta_{\mathrm{C}} 125.20$ and $\delta_{\mathrm{C}} 144.80$, suggesting the presence of a 3-methylpenta-2,4-dienoic acid moiety. This moiety was confirmed by the HMBC cross peaks (fig. 2) from $\mathrm{H}-2$ to $\mathrm{C}-1, \mathrm{C}-6, \mathrm{C}-4$, from $\mathrm{H}-6$ to $\mathrm{C}-2, \mathrm{C}-3, \mathrm{C}-4$ and from $\mathrm{H}-4$ to $\mathrm{C}-5, \mathrm{C}-2$, C-6. In the ${ }^{1} \mathrm{H}$ and ${ }^{13} \mathrm{C}$ NMR spectra, the signals of two tertiary methyl groups, two methylenes, one oxymethine, one oxymethylene, two oxygenated quaternary carbons, and one quaternary carbon were attributable to a bicyclohexane ring. This suggestion was proved by the HMBC correlations from $\mathrm{H}-9$ ' to C-4', C-5', C-6', C-8', from H-10' to C-1', C-2', C-8', from H-2' to C-1', C-3', C-4', C-8', C-10', from H-4' to C-2', C-6', C-8', C-9' and from H-6' to C-1', C-4', C-5', C-8', C-9'. Moreover, the signals of a $\beta$-D-glucopyranosyl unit were observed at $\delta_{\mathrm{H}} 4.38(1 \mathrm{H}, \mathrm{d}, J=$ 
$8.0 \mathrm{~Hz}, \mathrm{H}-1 ") / \delta_{\mathrm{C}} 103.13, \delta_{\mathrm{H}} 3.17(1 \mathrm{H}, \mathrm{t}, J=8.5 \mathrm{~Hz}, \mathrm{H}-2 ") / \delta_{\mathrm{C}} 75.13, \delta_{\mathrm{H}} 3.37(1 \mathrm{H}, \mathrm{m}, \mathrm{H}-3 ") / \delta_{\mathrm{C}}$ $78.10, \delta_{\mathrm{H}} 3.31(1 \mathrm{H}, \mathrm{m}, \mathrm{H}-4 ") / \delta_{\mathrm{C}} 71.68, \delta_{\mathrm{H}} 3.30(1 \mathrm{H}, \mathrm{m}, \mathrm{H}-5 ") / \delta_{\mathrm{C}} 77.97, \delta_{\mathrm{H}} 3.67(1 \mathrm{H}, \mathrm{dd}, J=4.0$, $11.5 \mathrm{~Hz}, \mathrm{H}-6 " \mathrm{a}), \delta_{\mathrm{H}} 3.89(1 \mathrm{H}, \mathrm{d}, J=11.5 \mathrm{~Hz}, \mathrm{H}-6 " \mathrm{~b}) / \delta_{\mathrm{C}} 62.78$. The long-range correlations between $\mathrm{H}-1$ " and C-3', and between $\mathrm{H}-5$ and C-1', C-5', C-8' indicated that the sugar linked at C-3' and the 3-methyl-penta-2,4-dienoic acid moiety was attached to C-8'. On the basis of the results described above and comparison of the NMR data with those in literature, the structure of 2 was determined to be $\left(1^{\prime} R, 3^{\prime} S, 5^{\prime} R, 8^{\prime} S, 2 E, 4 E\right)$-dihydrophaseic acid 3'- $O$ - $\beta$-D-glucopyranoside [6].

Table 2. The NMR data of compound 2.

\begin{tabular}{|c|c|c|c|c|c|c|c|}
\hline $\mathbf{C}$ & ${ }^{{ }^{*}} \boldsymbol{\delta}_{\mathbf{C}}{ }^{\mathbf{a}}$ & $\boldsymbol{\delta}_{\mathbf{C}}{ }^{\mathbf{a}, \mathbf{b}}$ & $\boldsymbol{\delta}_{\mathbf{H}}{ }^{\mathbf{a}, \mathbf{c}}$ & $\mathbf{C}$ & ${ }^{{ }^{*}} \boldsymbol{\delta}_{\mathbf{C}}{ }^{\mathbf{a}}$ & $\boldsymbol{\delta}_{\mathbf{C}}{ }^{\mathbf{a}, \mathbf{b}}$ & $\boldsymbol{\delta}_{\mathbf{H}}{ }^{\mathbf{a}, \mathbf{c}}$ \\
\hline 1 & nd & 177.20 & - & $6^{\prime}$ & 77.2 & 77.15 & $\begin{array}{c}3.76(1 \mathrm{H}, \mathrm{m}) \\
3.82(1 \mathrm{H}, \mathrm{m})\end{array}$ \\
\hline 2 & 126.9 & 125.20 & $5.85(1 \mathrm{H}, \mathrm{br} \mathrm{s})$ & $8^{\prime}$ & 83.4 & 83.29 & - \\
\hline 3 & 143.3 & 144.80 & - & $9^{\prime}$ & 19.9 & 19.73 & $1.18(3 \mathrm{H}, \mathrm{s})$ \\
\hline 4 & 131.2 & 132.63 & $7.88(1 \mathrm{H}, \mathrm{d}, 16.0)$ & $10^{\prime}$ & 16.5 & 16.37 & $0.95(3 \mathrm{H}, \mathrm{s})$ \\
\hline 5 & 132.9 & 132.02 & $6.37(1 \mathrm{H}, \mathrm{d}, 16.0)$ & $1^{\prime \prime}$ & 103.3 & 103.13 & $4.38(1 \mathrm{H}, \mathrm{d}, 8.0)$ \\
\hline 6 & 20.8 & 20.82 & $2.03(3 \mathrm{H}, \mathrm{s})$ & $2^{\prime \prime}$ & 75.2 & 75.13 & $3.17(1 \mathrm{H}, \mathrm{t}, 8.5)$ \\
\hline $1^{\prime}$ & 49.5 & 49.20 & - & $3^{\prime \prime}$ & 78.2 & 78.10 & $3.37(1 \mathrm{H}, \mathrm{m})$ \\
\hline $2^{\prime}$ & 43.0 & 42.88 & $\begin{array}{c}1.81(1 \mathrm{H}, \mathrm{m}) \\
2.00(1 \mathrm{H}, \mathrm{dd}, 7.0,13.5)\end{array}$ & $4^{\prime \prime}$ & 71.8 & 71.68 & $3.31(1 \mathrm{H}, \mathrm{m})$ \\
\hline $3^{\prime}$ & 74.2 & 74.00 & $4.27(1 \mathrm{H}, \mathrm{m})$ & $5^{\prime \prime}$ & 78.1 & 77.97 & $3.30(1 \mathrm{H}, \mathrm{m})$ \\
\hline $4^{\prime}$ & 42.9 & 42.82 & $\begin{array}{c}1.82(1 \mathrm{H}, \mathrm{m}) \\
2.22(1 \mathrm{H}, \mathrm{dd}, 7.0 ; 13.5)\end{array}$ & $6^{\prime \prime}$ & 62.9 & 62.78 & $3.67(1 \mathrm{H}, \mathrm{dd}, 4.0 ; 11.5)$ \\
\hline $5^{\prime}$ & 87.7 & 87.62 & - & - & - & - & $3.89(1 \mathrm{H}, \mathrm{d}, 11.5)$ \\
\hline
\end{tabular}

${ }^{a}$ recorded in $\mathrm{CD}_{3} \mathrm{OD},{ }^{b} 125 \mathrm{MHz},{ }^{c} 500 \mathrm{MHz}$, nd: Not detected.

${ }^{\#} \delta_{C}$ of $\left(I^{\prime} R, 3^{\prime} S, 5^{\prime} R, 8^{\prime} S, 2 E, 4 E\right)$-dihydrophaseic acid 3'-O- $\beta$-D-glucopyranoside[6]

Compounds 3-5 were identified as 3-methylbutan-1-ol $\beta$-D-glucopyranoside (3)[7], astragalin (4) [8] and daucosterol (5) [9] by comparing their NMR spectral data with those of reported in literature.

\section{CONCLUSION}

The phytochemical investigation of the seeds of Scaphium macropodum (Miq.) Beumée resulted in the isolation of five known compounds including \{carbonylbis[imino(6-methyl-3,1phenylene)] \}bis[carbamic acid] dimethyl ester (1), $\left(1^{\prime} R, 3^{\prime} S, 5^{\prime} R, 8^{\prime} S, 2 E, 4 E\right)$-dihydrophaseic acid 3'-O- $\beta$-D-glucopyranoside (2), 3-methylbutan-1-ol $\beta$-D-glucopyranoside (3), astragalin (4) and daucosterol (5). Their structures were identified by comparison of their spectroscopic data with those reported in the literature. This is the first report for the isolation of compounds $\mathbf{1 - 3}$ from this species. 
Acknowledgements. This work was financially supported by a Vietnam national project of the Tay Nguyen 3 Program (code: TN3/T14). The authors are grateful to Institute of Chemistry, VAST for measuring NMR spectra.

\section{REFERENCES}

1. Vo Van Chi - Dictionary of Vietnamese medicinal plants, Vol. 1, Publishing House Medicine, Ha Noi, 2012, 1367-1368.

2. Al Muqarrabun L. M., Ahmat N., Aris S. R., Shamsulrijal N., Baharum S. N., Ahmad R., Rosandy A. R., Suratman M. N. \& Takayama H. - A new sesquiterpenoid from Scaphium macropodum (Miq.) Beumee, Nat. Prod. Res. 28 (2014) 597-605.

3. Wang R. F., Yang X. W., Ma C. M., Shang M. Y., Liang J. Y., Wang X., Cai S. Q., and Shoyama Y. - Alkaloids from the seeds of Sterculia lychnophora (Pangdahai), Phytochemistry 63 (2003) 475-478.

4. Wang R. F., Wu X. W., and Geng D. - Two cerebrosides isolated from the seeds of Sterculia lychnophora and their neuroprotective effect, Molecules 18 (2013) 1181-1187.

5. Zhao You-Xing, Li, Cheng-Sen Luo, Xiao-Dong Yi, Tie-Mei and Zhou Jun - Three New Urea Derivatives from Pliocene-Fossil Pinus armandii, Helvetica Chimica Acta 88 (2005) 325-329.

6. Ui Joung Youn, Jun Lee, Joo Won Nam, Yoo Jin Lee, and Eun-Kyoung Seo Identification of a New Isomer of Dihydrophaseic Acid 3'-O- $\beta$-D-Glucopyranoside from Nelumbo nucifera, Bull. Korean Chem. Soc. 32 (2011) 4083-4085.

7. Junichi Kitajima, Toru Ishikawa, Yasuko Tanaka - Water-Soluble Constituents of Fennel. Part 1. Alkyl Glycosides, Chem. Pharm. Bull. 46 (1998) 1643-1646.

8. Wang X. L., Wang N. L., Zhang Y., Gao H., Pang W. Y., Wong M. S., Zhang G., Qin L. and Yao X. S. - Effects of eleven flavonoids from the osteoprotective fraction of Drynaria fortunei (KUNZE) J. SM. on osteoblastic proliferation using an osteoblast-like cell line, Chem Pharm Bull (Tokyo) 56 (2008) 46-51.

9. Yoo Jong-Su, Ahn Eun-Mi, Bang Myun-Ho, Song Myoung-Chong, Yang Hye-Joung, Kim Dong-Hyun, Lee Dae-Young, Chung Hae-Gon, Jeong Tae-Sook, Lee Kyung-Tae, Choi Myung-Sook, Baek Nam-In - Steroids from the Aerial Parts of Artemisia princeps Pampanini, Korean journal of medicinal crop science 14 (2006) 273-277.

\section{TÓM TÁT}

\section{CÁC GLUCOSIDE VÀ DẪN XUẤT CỦA URE PHÂN LẬP TỬ QUẢ ƯƠI SCAPHIUM MACROPODUM (MIQ.) BEUMÉE}

Vũ Anh Tú ${ }^{1}$, Châu Ngọc Điệp ${ }^{1}$, Nguyễn Hữu Toàn Phan ${ }^{2, *}$, Nông Văn Duy², Nguyễn Thị Diệu Thuần, Nguyễn Văn Thanh ${ }^{1}$, Nguyễn Xuân Cường ${ }^{1}$, Nguyễn Hoài Nam ${ }^{1}$, Châu Văn Minh ${ }^{1}$

${ }^{1}$ Viện Hóa sinh biển (IMBC), Viện Hàn lâm Khoa học và Công nghệ Việt Nam (VAST), 18 Hoàng Quốc Việt, Cầu giấy, Hà Nội

${ }^{2}$ Viện Nghiên cứu Khoa học Tây Nguyên, VAST, 116 Xô Viết Nghệ Tĩnh, Đà Lạt

*Email:nhtphan@gmail.com 
Sử dụng các phương pháp sắc ký kết hợp, năm hợp chất \{carbonylbis[imino(6-methyl-3,1phenylen)] \}bis[carbamic acid] dimethyl ester (1), (1'R,3'S,5' $\left.R, 8^{\prime} S, 2 E, 4 E\right)$-dihydrophaseic acid 3'$O$ - $\beta$-D-glucopyranoside (2), 3-methylbutan-1-ol $\beta$-D-glucopyranoside (3), astragalin (4) và daucosterol (5) đã được phân lập từ cặn chiết metanol của quả ươi - Scaphium macropodum (Miq.) Beumée. Cấu trúc hóa học của các hợp chất được xác định bằng các phương pháp phổ cộng hưởng từ hạt nhân và phổ khối lượng kêt hợp so sánh với các số liệu phổ đã được công bố. Các hợp chất 1-3 lần đầu tiên được phân lập từ cây ươi.

Tù khóa: Scaphium macropodum, Sterculiaceae, glucosides, urea derivative. 